\title{
LIDAR-EQUIPPED UAV FOR BUILDING INFORMATION MODELLING
}

\author{
D. Roca ${ }^{\text {a }}$ J. Armesto ${ }^{\text {a } *, ~ S . ~ L a g u ̈ e l a ~}{ }^{\text {a }}$ L. Díaz-Vilariño ${ }^{a}$ \\ a ETSE Minas, University of Vigo, Campus Lagoas-Marcosende, 36310, Vigo, Spain - (davidroca, julia, susiminas, lucia)@uvigo.es
}

Commission V, WG V/3

KEY WORDS: UAV, Lidar, GPS, point clouds, buildings, BIM

\begin{abstract}
:
The trend to minimize electronic devices in the last decades accounts for Unmanned Airborne Vehicles (UAVs) as well as for sensor technologies and imaging devices, resulting in a strong revolution in the surveying and mapping industries. However, only within the last few years the LIDAR sensor technology has achieved sufficiently reduction in terms of size and weight to be considered for UAV platforms. This paper presents an innovative solution to capture point cloud data from a Lidar-equipped UAV and further perform the 3D modelling of the whole envelope of buildings in BIM format. A mini-UAV platform is used (weigh less than $5 \mathrm{~kg}$ and up to $1.5 \mathrm{~kg}$ of sensor payload), and data from two different acquisition methodologies is processed and compared with the aim at finding the optimal configuration for the generation of 3D models of buildings for energy studies
\end{abstract}

\section{INTRODUCTION}

\subsection{Motivations}

Recent progress in aerospace technology is evolving towards the use of Unmanned Aerial Vehicles (UAV), especially focusing on their miniaturization and lightening. In the geomatics field, these vehicles are being increasingly used given their capacity to survey difficult-access areas, as well as acquiring data from large areas in a reduced amount of time.

Most works deal with the acquisition of photographic images (Haala et al, 2011) towards the generation of Geographic Information Systems, GIS, by applying photogrammetric techniques. Other approaches have been done by extending the use of satellite sensors for study of land uses and vegetation to the performance of the same studies from UAV platforms (Lagüela et al, 2013), and also energy efficiency in cities (Previtali et al, 2013). Last, some works have dealt with the use of Lidar sensors for the generation of plans of buildings for indoor navigation ( $\mathrm{He}$ et al, 2008) and autonomous missions (Bachrach et al, 2009).

However, given the flexibility of UAVs, these platforms appear as optimal solutions for building surveying, being able to reach the most inaccessible parts of buildings: upper part of the envelope and roof, that are missed in acquisitions performed with a terrestrial approach (Fruh \& Zakhor, 2003). In addition, UAV provide more detailed information than common surveying flights due to the capacity of these platforms of flying at lower heights, providing information of the vertical walls and not only of the horizontal planes (Gerke \& Xiao, 2014).

The main drawback of UAV platforms is their small payload, which restricts the variety of sensors to integrate. For this reason, flights with surveying purposes mainly used photographic or video cameras (Nex \& Remondino, 2014), although other RGB-D sensors can be find in literature (Lange, 2012). However, laser scanners are recognized as the most efficient sensors for geometric data acquisition, both in terms of acquisition time and precision, consequently increasing the interest of their integration in these platforms. In order to fulfil the payload requirement, only light line-scanners or 2D laser scanners can be mounted in UAV platforms, making it necessary for 3D data acquisition the integration of positioning sensors, IMU and GPS (Wu et al., 2013).

In this work, a lidar-equipped UAV is presented for the generation of 3D point clouds of building envelopes, towards their use for building assessment. Since outdoor navigation is performed, UAV positioning is supported by the use of online DGPS correction. Regarding the two main advantages of UAVs: their nature of aerial platform and their capacity of flying at low heights and in small areas, two acquisition methodologies have been analysed in this work. First, a common aerial acquisition was developed, acquiring only information of a building roof and the floor; second, a lowheight flight was performed, acquiring information of both the façade of the building and the roof. In both cases, the precision of the 3D point cloud generated is analysed with the aim at checking whether precision fulfils the requirements for its application to energy studies. As different example applications, semantic 3D models are generated from the point clouds, reaching LoD2 (Level of Detail 2).

The paper is organized as follows: Section 2 presents the UAV and the integrated sensors, as well as the acquisition methodologies and the procedure for the generation of 3D point clouds. Section 3 analyses the results and their application to the generation of different semantic 3D models. Section 4 contains the conclusions reachable.

\section{METHODOLOGY}

\subsection{Materials}

The platform chosen for the aerial inspection unit has been provided by HiSystems GmbH. The model is an eight propeller copter, referred to as Okto XL. The basis of the copter consists on a frame of aluminium square tubes and carbon fibre base plates. The system is controlled by the Flight Control, which is a circuit board including a three axis accelerometer, a three axis 
gyroscope, and a pressure sensor, which are mainly used to calculate the height and orientation, improve the stability of the copter, and ease its control. What is more, the eight MK-3638 black brushless motors drive left and right by rotating APC SlowFly 12 × 3.8 propellers, and can be separately controlled by the Brushless Control, thus increasing the stability of the platform.

The Hokuyo UTM-30LX is a low cost laser sensor that has been chosen for the generation of 3D point clouds in this work, and it has been mounted on the aerial unit, held by an aluminium frame specially designed for this purpose. The Hokuyo sensor consists on a rotating mirror laser that measures up to 40.000 points per second in a range between 0.1 and 30 meters. The rotating frequency is $40 \mathrm{~Hz}$, that is, a profile is measured each $25 \mathrm{~ms}$. Attached to the laser, a low cost IMU is mounted to provide orientation and position measurements. The IMU is from Advanced Navigation, model Spatial; this sensor is a GPS aided inertial navigation system that provides measurements at $500 \mathrm{~Hz}$ frequency, with $2 \mathrm{~m}$ accuracy in horizontal position, 3 $\mathrm{m}$ accuracy in the vertical position, and an angular accuracy of $0.1^{\circ}$ for roll and pitch, and $0.5^{\circ}$ for heading. Regarding GPS, its accuracy in the determination of the coordinates is $2.5 \mathrm{~m}$, which is very low for the surveying of buildings.

For this reason, in this work the absolute position is given by a Trimble BD920 GNSS receiver that works in RTK mode, reaching accuracies of $0.008 \mathrm{~m}$ in horizontal coordinates, and $0.015 \mathrm{~m}$ in vertical coordinates, using satellites from GPS and GLONASS.

To power the control system and the sensors a Lipo 4S 2200 mAh $14.8 \mathrm{~V}$ battery and a voltage regulator have been used independent of the copter alimentation system.

Figure 1 shows the UAV platform equipped with all the sensors necessary for the 3D surveying of buildings.

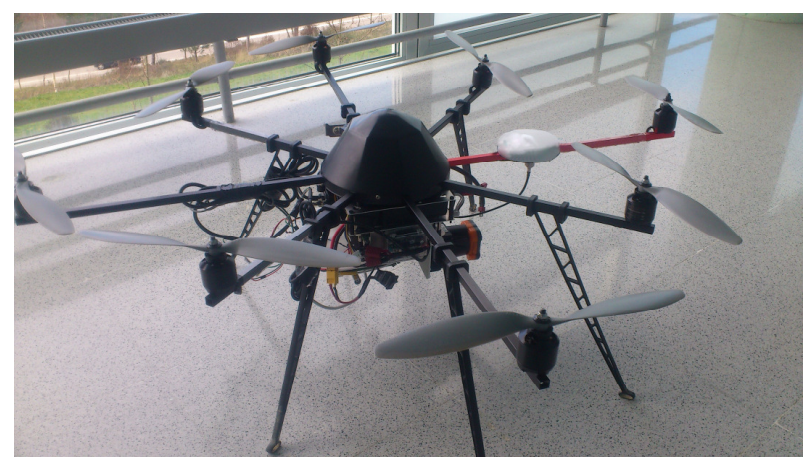

Figure 1. UAV platform equipped with 2D laser scanner Hokuyo and GPS.

\subsection{Data acquisition}

Data capture is performed through an Advantech PCM-9363 circuit board, which has an Intel Atom D525 processor with an USB connector for IMU and laser, Ethernet connector for GPS, a SATA connector for a Solid State Disk where data are stored and a wireless USB device for communication.

To operate the acquisition system a land laptop has been used. It is connected through its wireless device to the scan system by 802.11 communication protocol in $2.4 \mathrm{GHz}$ frequency band. The control of the data acquisition is made in real time, and includes the start capture, the correct record of data and the end of the acquisition process.

A house located in Galicia (Northwest of Spain) was selected as case of study. It is a detached house half surrounded by trees, consequently suffering their interference regarding satellite reception: the number of satellites detected was 5 .

With the aim at analyzing the different acquisition options available for the UAV platform, two different data acquisition methodologies were followed: the first data acquisition was based on the common surveying flights, following a trajectory over the building at higher flying heights and acquiring only zenithal information: roofs and floor. In this case of study, the flight was performed at $15 \mathrm{~m}$ height. The flight was performed perpendicular to the main façade, being the direction of the trajectory coincident with the depth of the building, as shown in figure 2 .

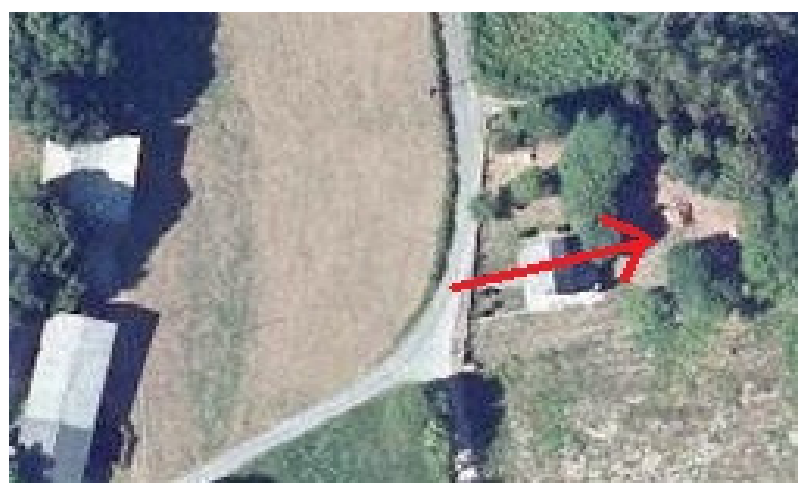

Figure 2. Flying path in Methodology 1.

The second data acquisition was performed looking at the maximization of the data acquired, surveying both the façade and the roof. For this reason, the flight was performed at lower height, $10 \mathrm{~m}$, and from $10 \mathrm{~m}$ distance to the façade, so that lateral information could also be acquired. In this case, the direction of the trajectory was parallel to the main façade, figure 3.

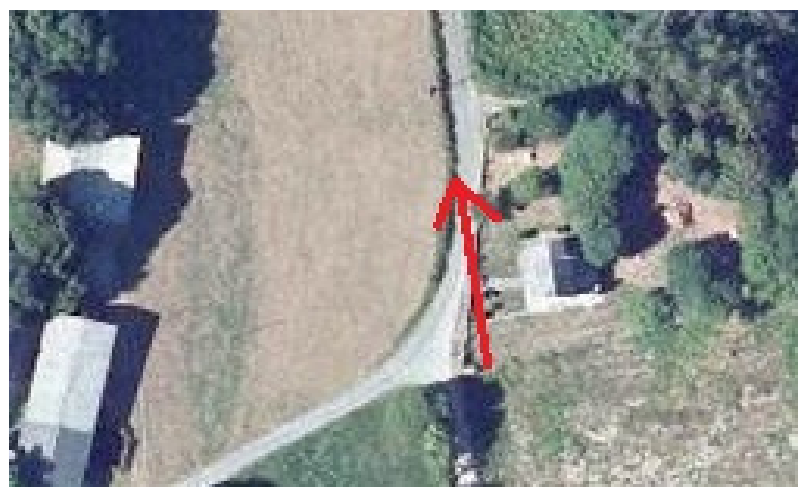

Figure 3. Flying path in Methodology 2.

Values of height and horizontal distance to the building are to be established for each building under study, regarding their geometry and the environment of their location, looking for accessibility. 


\subsection{Data processing}

Lidar data are hardware synchronized so that each scan of the Hokuyo laser has a position and orientation measurement associated. Given the different measuring frequencies of the sensors used, the procedure is the following: the IMU measures at $500 \mathrm{HZ}$ frequency, calculating the performed trajectory via the Kalman filter that is internally implemented in the sensor. Since the GPS provides a position value each second, it is used for correcting the drift of the calculated trajectory by the introduction of the GPS data in the Kalman filter.

Once the flying trajectory is determined, each $2 \mathrm{D}$ scan $(y, z$ coordinates of each point) from the Hokuyo is placed in its corresponding position, regarding the sensor synchronization per time; this way, each 2D scan acquires its $x$ coordinate (defining $x$ as the trajectory direction), so that the $3 \mathrm{D}$ model is generated.

Data processing is the same for the two flying methodologies analysed in this paper, and the results are shown in figures 4 and 5 .

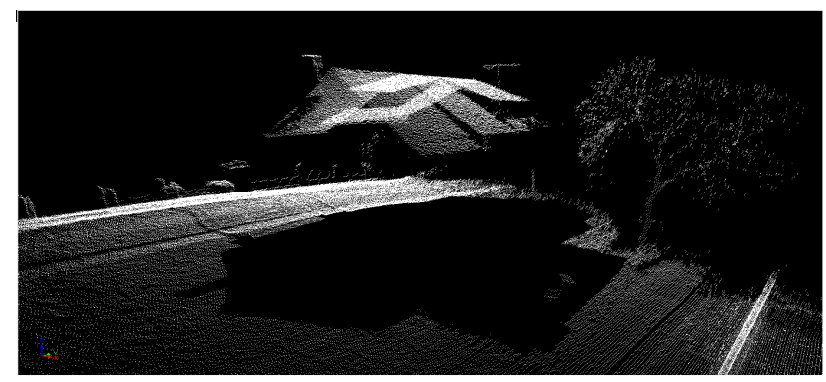

Figure 4. Example of point cloud acquired by the UAV system with 2D laser and GPS following methodology 1: roof acquisition.

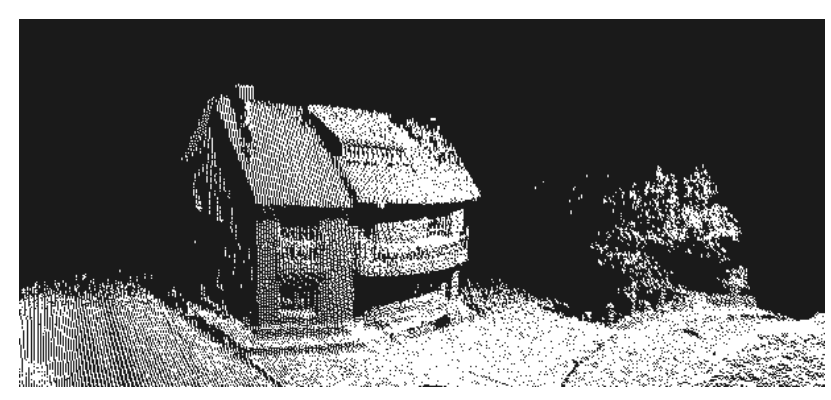

Figure 5. Example of point cloud acquired by the UAV system with 2D laser and GPS following methodology 2: combined acquisition of façade and roof.

\section{RESULTS}

\subsection{D point clouds}

The density of the point cloud obtained varies depending on the speed of the UAV. In the case of study, the flying speed was set at $2 \mathrm{~m} / \mathrm{s}$, so that a $2 \mathrm{D}$ scan is performed every $5 \mathrm{~cm}$. This speed leads to the measuring of all the existing elements in the building, since construction works with greater sizes.

In order to evaluate the methodology, the quality of each point cloud generated from data acquired with the different methodologies is determined through its comparison with a point cloud acquired with a terrestrial laser scanner, FARO Focus 3D X330. This laser scanner has $2 \mathrm{~mm}$ accuracy in the measurement of points, value which make it usable as reference for geometric measurements. The point cloud measured with FARO Focus laser scanner is shown in figure 6; in this case, each point also has its RGB value due to the availability of a photographic camera attached to the scanner.

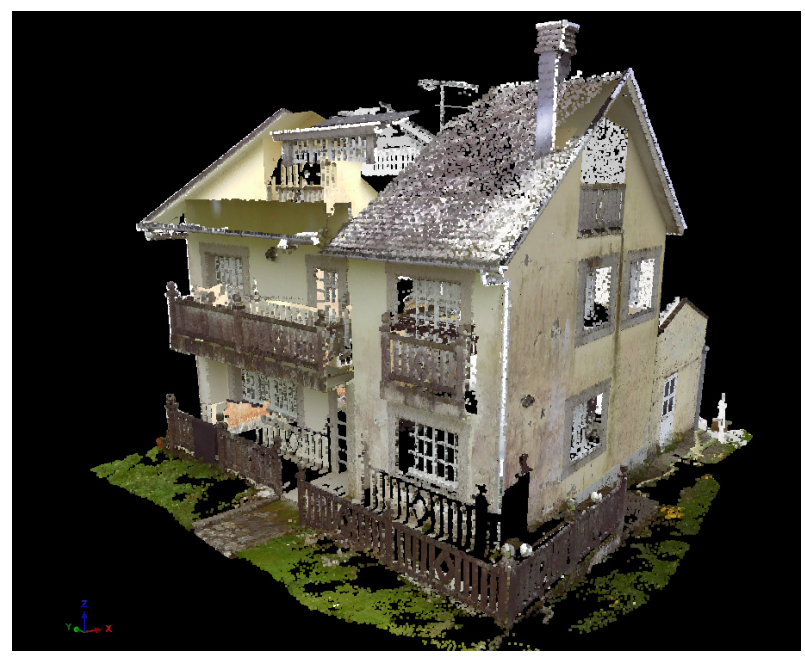

Figure 6. Reference point cloud, measured with the terrestrial laser scanner FARO Focus 3D X330.

Given that the volume of the building is the geometric key element for the generation of energy efficiency studies (determination of heating requirements, volume of inner air, air infiltration capacity), the quality of the point clouds generated with data acquired from the proposed aerial system is determined through their comparison with the reference values. The results of these comparison are shown in table 1.

\begin{tabular}{|l|c|c|c|c|c|}
\hline POINTCLOUD & \multicolumn{5}{|c|}{ MEASUREMENT } \\
\hline & HEIGHT & WIDHT & DEPTH & AREA & VOL \\
REFERENCE & 5.3 & 9.0 & 6.8 & 61.2 & 324.36 \\
METHOD. 1 & 5.8 & 9.5 & 7.7 & 73.2 & 424.27 \\
METHOD. 2 & 5.2 & 10.8 & 7.0 & 75.6 & 393.12 \\
\hline
\end{tabular}

Table 1. Geometric results obtained from the point clouds acquired with the FARO Focus (reference) and the different methodologies ( 1 for the zenithal methodology, 2 for the façade methodology)

Results show that the first flying methodology, acquiring information of the roof, presents a $30.8 \%$ deviation regarding the volume of the building, whereas the second flying methodology, which provides data of both the façade and the roof, has $21.2 \%$ deviation for the volume.

Results show that, in both cases, the greatest error appears in the flying direction: the depth of the building for the methodology 1 , and the width of the building for the methodology 2 . 


\subsection{Semantic 3D Modelling}

Given the different data acquired with the two flying methodologies tested, processing procedures towards an energy application differ between the two.

For the case of Methodology 1, where only information of the roof and the ground is acquired, a GIS 3D is generated, using the contours of the roof and extruding the footprint of the roof down to the ground. The result is shown in figure 7.
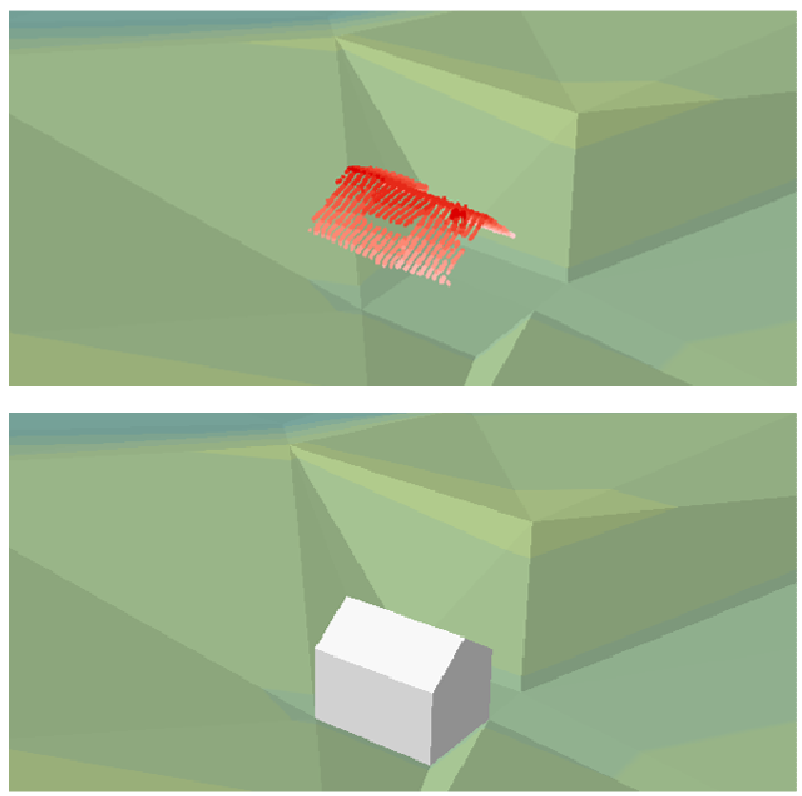

Figure 7. Point cloud acquired with Methodology 1 in its position on a Digital Terrain Model, DTM (up), and 3D model of the building, in GIS 3D format (down).

Regarding Methodology 2, the 3D point cloud generated presents information about the roof and the walls, allowing the performance of a segmentation procedure based on a curvature analysis and 3D region growing, presented in (Díaz-Vilariño et al, 2013), so that each surface is identified and defined. Surface intersection is performed for the computation of the coordinates of the points of each surface. These points are used in the definition of the geometry of the semantic 3D building model of the case of study using gbXML format, chosen due to its capabilities in energy software, figure 8 . With this methodology, the result is more detailed, with 4 façade surfaces, 4 roof surfaces (gable roof and 2 eaves, in the frontal and the back façades), and the floor surface.

Since the GPS provides global coordinates of the points, also the location and the orientation of the building are known, in such way that the results of the presented methodology are accurately geolocated.

\section{CONCLUSIONS}

In this paper, a preliminary approach is presented for the use of UAV platforms, copter-type, for the 3D surveying of buildings, towards the generation of semantic 3D models LoD2, with different capabilities depending on the acquisition methodology: in every case, results can be used for large-scale studies with little geometric detail, such as urban energy tendencies. Whereas the most detailed semantic 3D models are usable for individual energy-related studies, as natural lighting input, heating losses and requirements.

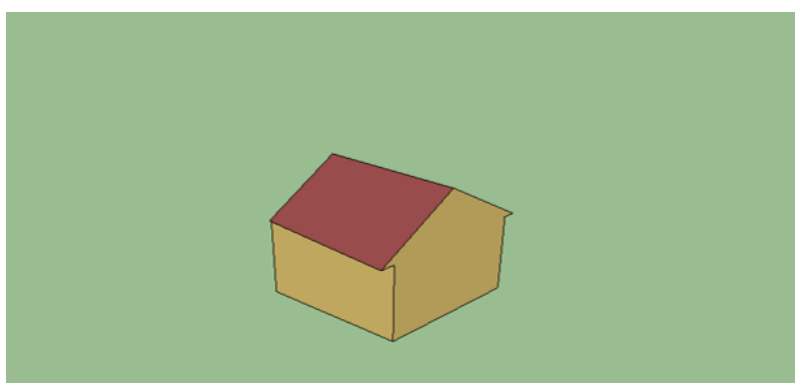

Figure 8. Semantic 3D model of the building, in gbXML format, generated from data acquired with flying methodology 2.

Two different flying approaches have been tested, with the aim at selecting the optimal methodology. In both approaches, the greatest deviation is found in the dimension coincident with the flying direction, showing that the main source of error is the computation of the trajectory. In those cases where tree coverage is not so important, satellite reception will be improved, consequently reducing the geometric deviation. Future work will deal with more severe cases, such as buildings in urban canyons or with more forest coverage, where GPS reception is worse and the trajectory depends mainly on the IMU measurements and the Kalman Filter.

Regarding the deviation values, the comparison of the two flying methodologies with the reference point cloud shows that the error in height is five times bigger for Methodology $1(9.4 \%$ versus $1.9 \%$ ), probably due to the higher flying height and the availability of data only from roof and floor, with no reference of the building façades. For this reason, under the same GNSS coverage, this preliminary approach determines that Methodology 2 is better for 3D model generation of buildings, given that the error is lower in the volumetric measurement, which is the most important geometric value for energy studies. What is more, Methodology 2 provides more detailed information of the building, being segmentable into the different existing surfaces: walls, roof, floor and eaves, consequently enriching the knowledge about the building and energy aspects such as shadow projections and natural lighting.

With respect to the application of the presented aerial system to the 3D surveying of buildings, the limitations of the generated model are established mainly by the sensor, since measurements with the Hokuyo are highly influenced by its maximum range: the closer to the range limit, the lower the precision. However, in case bigger buildings are interesting for their surveying, the same system would be applicable, with the only substitution of the laser sensor by a sensor with wider measurement range.

\section{ACKNOWLEDGEMENTS}

Authors would like to thank the Ministerio de Economía y Competitividad and CDTI (Gobierno de España) for the financial support given through projects (IPT2012-1092120000 and ITC-20133033) and human resources (FPU AP2009-1144 and FPU AP2010-2969). All the programs are partially financed by the Fondo Europeo para el Desarrollo Regional (FEDER). 


\section{REFERENCES}

Bachrach, A., He, R., Roy, N., 2009, Autonomous flight in unknown indoor environments, International Journal of Micro Air Vehicles, 1(4), pp. 217-228.

Díaz-Vilariño, L., Lagüela, S., Armesto, J., Arias, P., 2013, Semantic as-built 3D models including shades for the evaluation of solar influence on buildings, Solar Energy, 92, pp. 269-279.

Fruh, C., Zakhor, A., 2003, Constructing 3D city models by merging aerial and ground views, IEEE Computer Graphics and Applications, 23(6), pp. 52-61.

Gerke, M., Xiao, J., 2014, Fusion of airborne laserscanning point clouds and images for supervised and unsupervised scene classification, ISPRS Journal of Photogrammetry and Remote Sensing, 87, pp. 78-92.

Haala, N., Cramer, M., Weimer, F., Trittler, M., 2011, Performance test on UAV based photogrammetric data collection, IAPRS 38(1/C22), pp. 7-12.

He, R., Prentice, S., Roy, N., 2008, Planning in information space for a quadrotor helicopter in a GPS-denied environment, Proc. IEEE International Conference on Robotics and Automation, Los Angeles (USA).
Lagüela, S., Díaz-Vilariño, L., Roca, D., Armesto, J., 2013, Aerial thermography from low-cost UAV for the generation of thermographic digital terrain models, AITA Abstract Book, Torino (Italy), pp. 77-80.

Lange, S., Sünderhauf, N., Neubert, P., Drews, S., Protzel, P., 2012, Autonomous corridor flight of a UAV using a low-cost and light-weight RGB-D camera, International Symposium on Autonomous Minirobots for Research and Edutainment, pp. 183-192.

Nex, F., Remondino, F., 2014, UAV for 3D mapping applications: A Review, Applied Geomatics, 6(1), pp. 1-15.

Previtali, M., Barazzetti, L., Brumana, R., Roncoroni, F., 2013, Thermographic analysis from UAV platforms for energy efficiency retrofit applications, Journal of Mobile Multimedia, 9(1-2), pp. 66-82.

Wu, J., Li, Q., He, M., Zhang, F., 2013, A terrain model generation method based on 2D plan laser scanner for micro UAV autonomous flight, International Conference on Information Technology and Software Engineering, 211, pp. 215-225. 